\title{
Evaluación del Desempeño de Buriles con Recubrimiento Monocapas de Nitruro de Hafnio en el Proceso de Mecanizado
}

\author{
John H. Navarro-Devia( ${ }^{(1)}$, Willian A. Aperador ${ }^{(1) *}$ y Arnoldo Delgado(2). \\ (1) Grupo de Investigación Volta, Facultad de Ingeniería, Universidad Militar Nueva Granada, \\ Carrera 11 No.101-80, Bogotá-Colombia (e-mail: jhnavd@gmail.com; g.ing.materiales@gmail.com). \\ (2) Grupo de Investigación Energía Alternativa, Facultad de Ingeniería, Universidad Militar Nueva \\ Granada, Carrera 11 No. 101-80, Bogotá-Colombia.
}

* Autor a quien debe ser dirigida la correspondencia

Recibido Jul. 3, 2015; Aceptado Ago. 21, 2015; Versión final Oct. 13, 2015, Publicado Feb. 2016

\section{Resumen}

Recubrimientos monocapa de nitruro de hafnio fueron depositados en buriles de acero rápido, mediante técnica de deposición física en fase vapor mediante pulverización con magnetrón (magnetrón sputtering). Se usó buriles sin recubrir como sistemas de referencias, en el mecanizado por arranque de viruta del acero AISI1020, midiendo las temperaturas generadas. Se caracterizó la resistencia al desgaste de las herramientas evaluándolas mediante microscopia electrónica de barrido, y las superficies obtenidas en el acero mecanizado mediante un rugosímetro. Se evidenció que el uso de recubrimientos monocapa de nitruro de hafnio en buriles de acero rápido disminuye el desgaste adhesivo de la herramienta y la transferencia de energía generada durante el proceso de corte. Además, el recubrimiento ofrece ventajas en su aplicación en el corte por arranque de viruta de metales ferrosos, al aumentar la vida útil de la herramienta, mejorar la calidad del producto, y disminuir los tiempos y costos del proceso.

\section{Performance Evaluation of Monolayer Hafnium Nitride Coated Tool for Cutting}

\begin{abstract}
Hafnium nitride monolayer coatings were deposited as thin films on high-speed steel by magnetron sputtering physical vapor deposition. Uncoated high-speed steels ASSAB 17 were used as reference systems, for machining on AISI1020 steel by chip removal, measuring the temperature during the process. Wear resistance of the tools was characterized by using scanning electron microscopy, and through of the surfaces of the steel using a roughness tester. It was evidenced that the use of monolayer hafnium coatings on uncoated high-speed steel reduces the adhesive wear of the tool and the energy transfer during the cutting process. Also, hafnium nitride coating offers advantages in its application to chip removal process of ferrous metals, by increasing the life of the tool, improving the quality of the work piece, and reducing cutting process time and cost.
\end{abstract}

Keywords: wear;monolayer;machining;cutting tools;hafnium nitride 


\section{INTRODUCCIÓN}

Uno de los principales problemas presentes en la industria mundial, sea automotriz, metalmecánica e incluso la petrolera, es el desgaste de sus herramientas de trabajo lo que conlleva a perdidas en calidad, cantidad de producción y sobrecosto en los procesos de manufactura. Con el fin de mejorar las propiedades mecánicas tales como dureza, resistencia al desgaste (Bobzin et al., 2014), aumento de la resistencia a la corrosión y diminución del coeficiente de fricción, se han realizado procesos llamados recubrimientos duros (Capote Rodriguez et al., 2015), que permiten que estas piezas puedan ser utilizadas en aplicaciones específicas como en las industrias metalmecánica, papelera, de transformación de materiales poliméricos y alimentaria, entre otras (Nieto et al., 2010). Los recubrimientos se han venido utilizando comercialmente para prolongar la vida útil de elementos industriales como las herramientas de corte y componentes de máquinas, manteniendo una gran acogida y utilización de forma industrial (Davies et al., 2007). La aplicación de recubrimientos duros en herramientas de bajo costo busca mejorar las propiedades mecánicas, aumentando la vida útil de estas y reduciendo costos derivados de las paradas para re afilado o reposición de la herramienta, sin disminuir la calidad de las piezas obtenidas en el proceso de mecanizado, e incluso disminuir el impacto ambiental (Åstrand et al., 2004; Klocke et al., 2006; Bouzakis et al., 2009 y Smith et al., 1997). Algunos procesos de mecanizado se ejecutan con velocidades de corte altas y profundidades de corte incrementadas, lo que puede causar el problema del desgaste adhesivo de la herramienta, generando una disminución de su vida útil y problemas con los acabados superficiales y precisión de las piezas trabajadas (Pawade et al., 2008). La utilización de herramientas con recubrimientos reduce la velocidad de desgaste adhesivo lo cual concluye en menores inconvenientes para lograr el control dimensional y superficial de los productos (Kant y Sangwan, 2014). Las herramientas de corte como buriles, brocas y fresas, son utilizadas en los procesos de fabricación por medio del mecanizado y el arranque de viruta debido a que por ser elaboradas por materiales con buenas propiedades permiten realizar estos desbastes y moldear las piezas (Nayak et al., 2014 y Vogtel et al., 2014). Sin embargo estas herramientas de corte van sufriendo el desgaste adhesivo superficial por las altas velocidades de trabajo, por las temperaturas alcanzadas durante la fricción y el arranque de viruta (Palanikumar y Davim, 2007).

El propósito del trabajo es la evaluación de la vida útil de buriles de acero rápido, recubierto por un sistema de tipo monocapa de HfN, obteniendo los resultados frente al desgaste adhesivo de estas herramientas debido a su uso prolongado, realizando pruebas en un centro de mecanizado que permitan identificar dicho comportamiento. Estos resultados serán comparados con los presentados por una herramienta del mismo material sin recubrimiento y que será usado en iguales condiciones y con los mismos parámetros del material recubierto.

\section{METODOLOGÍA}

\section{Deposición del recubrimiento}

Los recubrimientos de monocapas de HfN se depositaron sobre buriles de acero rápido ASSAB 17 de $3 / 8 \mathrm{X}$ 3 ", los cuales fueron desengrasados por ultrasonido en una secuencia de 15 minutos de etanol y acetona. Los recubrimientos se obtuvieron mediante la técnica del magnetrón sputtering. Para la deposición de los recubrimientos se utilizaron blancos de 4 pulgadas de diámetro de hafnio con una pureza del 99,9\%. Los parámetros de crecimiento se detallan en la Tabla 1. El sistema consta de cuatro cañones tipo magnetrón sputtering de cuatro pulgadas de diámetro; tres fuentes de radio frecuencia $(13,56 \mathrm{MHz})$, sistema de bombeo turbomolecular, medidores de alta y baja presión, controladores de presión; sistema de calentamiento por radiación desde temperatura ambiente hasta $400^{\circ} \mathrm{C}$, fluxómetros digitales para cuatro gases. Antes de la deposición, la cámara de vacío fue evacuada, por medio de la bomba turbomolecular a una presión base de $2,3 \times 10^{-5}$ con el objetivo de reducir los efectos del aire residual. Al interior de la cámara, los buriles fueron sometidos durante 15 minutos a un voltaje bias de $-400 \mathrm{~V}$ (r.f.) con una potencia de $60 \mathrm{~W}$ (r.f) en el plasma de argón (Ar) para remover cualquier capa de óxido. Sobre los buriles de acero se depositó una capa intermedia de Hf para las películas de HfN, esto se llevó a cabo bajo deposición continua para el $\mathrm{Hf}$ a una potencia r.f. de $350 \mathrm{~W}$ y $400 \mathrm{~W}$, en una atmosfera de argón por 5 minutos (Staia et al., 2006; Beake y Fox-Rabinovich, 2014). b. El espesor de los recubrimientos fue obtenido mediante un perfilómetro DEKTAK 8000 con un diámetro de punta de $12 \mathrm{~mm}$ a una longitud de barrido entre $1000 \neg 1200$ milimetros. Para la muestra de Nitruro de hafnio, el espesor fue de 1,20 micrómetros.

\section{Proceso de Corte}

Se realizó el mecanizado por arranque de viruta, del acero AISI 1020 en condiciones de ausencia de lubricación, en un centro de control numérico (CNC), midiendo las temperaturas generadas durante el proceso en el Flanco de desgaste borde de corte del Buril, en el punto del acero diametralmente opuesto a la ubicación del buril, y en viruta la desprendida, utilizando la herramienta con y sin recubrimiento, con los parámetros de corte definidos en la Tabla 2, constantes para todas las pruebas (Mandal et al.; 2011). 
Tabla 1: Parámetros de deposición utilizados para la obtención de los recubrimientos monocapas de HfN sobre los buriles.

\begin{tabular}{|l|l|}
\hline \multicolumn{1}{|c|}{ Parámetro } & \multicolumn{1}{c|}{ Valor } \\
\hline Densidad de potencia & $4,5 \mathrm{~W} / \mathrm{cm}$ \\
\hline Relación $\mathrm{N}_{2} / \mathrm{Ar}$ & $20 / 80 \%$ \\
\hline Flujo $\mathrm{N}_{2}$ & $10 \mathrm{sccm}$ \\
\hline Presión de trabajo & $1,2^{*} 10^{-2} \mathrm{mbar}$ \\
\hline Voltaje de polarización & $-30 \mathrm{~V}$ \\
\hline Tasa de deposición $(\mathrm{nm} / \mathrm{h})$ & 385 \\
\hline
\end{tabular}

Tabla 2: Parámetros del proceso de corte por arranque de viruta.

\begin{tabular}{|l|l|}
\hline \multicolumn{1}{|c|}{ Parámetro } & \multicolumn{1}{c|}{ Valor } \\
\hline Velocidad de rotación Husillo & $500 \mathrm{rpm}$ \\
\hline Velocidad de avance de la herramienta & $0,25 \mathrm{~mm} / \mathrm{rev}$ \\
\hline Penetración Diametral & $2,0 \mathrm{~mm}$ \\
\hline Longitud de la varilla & $30 \mathrm{~cm}$. \\
\hline Longitud del corte & $10 \mathrm{~cm}$. \\
\hline Diámetro de la varilla & $1 / 2 "$ \\
\hline
\end{tabular}

En la figura 1, se observa el montaje realizado para medir la temperatura mediante un sensor infrarrojo con un lente de convergencia que permite tener una medición sobre una área de $0.8 \mathrm{~mm}$ a $10 \mathrm{~mm}$ de distancia, con las especificaciones detalladas en la Tabla 3.

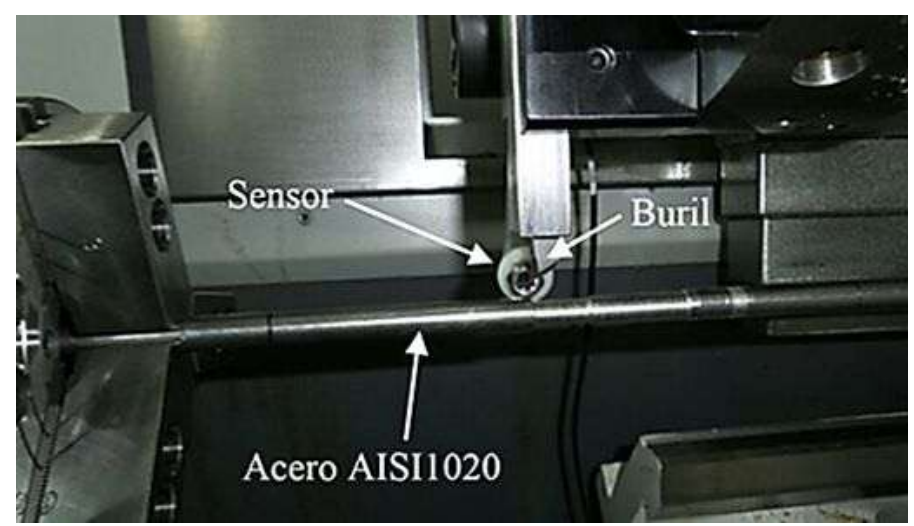

Fig. 1: Montaje realizado para la medición de temperatura.

Tabla 3: Parámetros técnicos sensor de temperatura PSC-CS Process Sensors.

\begin{tabular}{|l|l|}
\hline Característica & Especificación Técnica \\
\hline Tipo & Infrarrojo (Pirómetro) \\
\hline Rango de Temperatura & $-40^{\circ} \mathrm{C}$ a $1030^{\circ} \mathrm{C}$ \\
\hline Resolución Espacial & $15: 1$ resolución óptica \\
\hline Resolución de Tiempo & $25 \mathrm{~ms}$ \\
\hline Configuración & Por USB y Software DAQ \\
\hline Incertidumbre & $+/-1,5^{\circ} \mathrm{C}$ o $+/-1,5 \%$ \\
\hline Longitud de Onda & $8-14 \mu \mathrm{m}$ \\
\hline Rango Emisividad & Ajustable \\
\hline Accesorios & Lente Convergente \\
\hline Objetivo & $0.8 \mathrm{~mm}$ a $10 \mathrm{~mm}$ de distancia \\
\hline Software adquisición de Datos & LabView $2013 \mathrm{SP} 1$ \\
\hline Datos por segundo & 52 \\
\hline
\end{tabular}


En la figura 2, se observa el esquema del sensor y su conexión mediante USB, el cuál envía un valor numérico al equipo de cómputo; utilizando el software de adquisición de datos LabView, figura 3, esta información es interpretada, visualizada y actualizada a una tasa de 52 datos por segundos; además es almacenado en un archivo plano para posterior análisis de los datos.

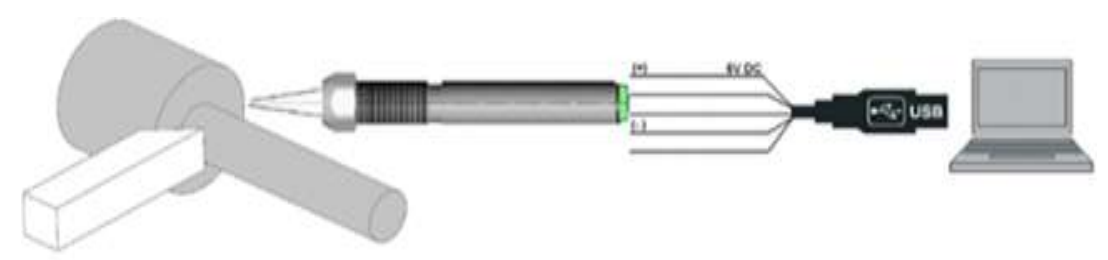

Fig. 2: Esquema de la medición de temperatura.

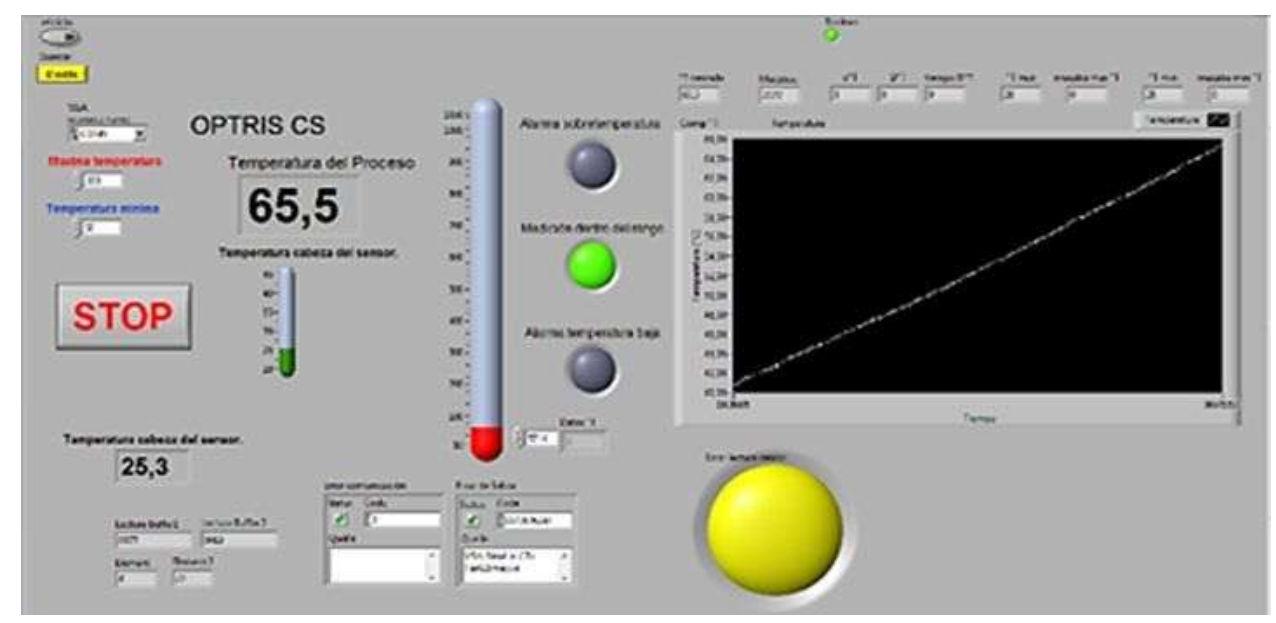

Fig. 3: Interfaz Gráfica en Labview medición de temperatura.

Luego se realizó el pulido de las probetas AISI 1020, mecanizadas anteriormente con la herramienta sin recubrimiento. En la primer corte se utilizó una velocidad de rotación de la copa $500 \mathrm{rpm}$, velocidad de avance de la herramienta $0.08 \mathrm{~mm} / \mathrm{rev}$; penetración diametral de $0.25 \mathrm{~mm}$, y al tener el CNC un sistema de control de velocidad, durante este proceso la herramienta presentaba una velocidad de $40 \mathrm{~mm} / \mathrm{min}$. Inmediatamente finalizada esta operación, se repetía el pulido de la pieza modificando los parámetros de operación, ahora con: velocidad de rotación de la copa $1000 \mathrm{rpm}$, velocidad de avance de la herramienta $0,01 \mathrm{~mm} / \mathrm{rev}$, penetración diametral de $0,10 \mathrm{~mm}$, y $10 \mathrm{~mm} / \mathrm{min}$ velocidad de la herramienta.

Para las observaciones y mediciones de desgaste de los buriles, con y sin recubrimiento, se empleó un microscopio electrónico de barrido (MEB), Jeol NeoScope JCM 5000. Para poder medir las superficies de los materiales en el grado de pulido e identificar el grado de irregularidad se utilizó un rugosímetro PCE-RT 1200 , este equipo detecta la rugosidad de superficies, al ser un dispositivo dotado de un palpador de diamante que desplaza una cierta longitud sobre el material, aunque podría haberse evaluado por inspección en microscopio óptico y electrónico (Alves et al., 2011). Se determinó una serie de parámetros que aportan el valor numérico de la rugosidad de acuerdo con las reglas de normalización, a las que este tipo de sistemas de medida están sujetos, estos parámetros, definidos como Ra y Rz (Sai Chaitanya et al., 2011).

\section{RESULTADOS Y DISCUSIÓN}

\section{Difracción de rayos $X$}

En la figura 4, se muestra el patrón de difracción de rayos $X$ de la película depositada HfN; el patrón de difracción de rayos $X$ presenta una estructura cúbica donde el pico más fuerte en $2 \theta=34,40^{\circ}$ corresponde al plano (111) HfN, lo que indica un crecimiento de textura luz a lo largo de esta orientación. Los otros picos débiles corresponden a difracciones de (200) y (220) planos de la cúbico (FCC) de tipo $\mathrm{NaCl}$. También se evidencia un pico de Bragg en $2 \theta=34.26^{\circ}$ asociado con la capa amortiguadora $\mathrm{Hf}$ en planos (100).

\section{Temperaturas del Proceso}

En las figuras 5,6 y 7 , se muestran las temperaturas de la operación de corte y los parámetros correspondientes al promedio de las pruebas realizadas, en las Tablas 4,5 y 6 ; identificando diferencias en 
los condiciones evaluadas, con el uso de los buriles con recubrimiento de nitruro de Hafnio, en comparación con el comportamiento del buril convencional sin recubrir. La temperatura promedio de la herramienta sin recubrir es mayor a $50^{\circ} \mathrm{C}$, pero al realizar el corte con la herramienta recubierta esta temperatura promedio se reduce hasta en $17^{\circ} \mathrm{C}$, evidenciando disminución de la energía transferida a la herramienta causado por la reducción de la fricción por el efecto del recubrimiento (Young, 1996). Además la herramienta recubierta presenta una tasa de cambio de temperatura, no superior a $3,0^{\circ} \mathrm{C} / \mathrm{min}$, en contraste a los $7,4^{\circ} \mathrm{C} / \mathrm{min}$ del buril sin recubrir, reflejando un aumento en la estabilidad de la transferencia de energía durante el transcurso del proceso; por lo cual la temperatura de estabilización del proceso disminuye de $68^{\circ} \mathrm{C}$ a $40^{\circ} \mathrm{C}$, debido a la monocapa de nitruro de hafnio depositada sobre el buril. Esto genera una reducción tanto de la energía transferida al sistema (Buril-acero-viruta) como del desgaste adhesivo, indicando que la energía total transferida durante al proceso, y el respectivo desgaste generado, será menor (El Hakim et al., 2015). También se identifica que sobre la herramienta recubierta se ejerce una menor fuerza en el arranque de viruta, por lo cual la temperatura máxima detectada en la herramienta, pasó de $81^{\circ} \mathrm{C}$ a $48^{\circ} \mathrm{C}$ con el recubrimiento de HfN.

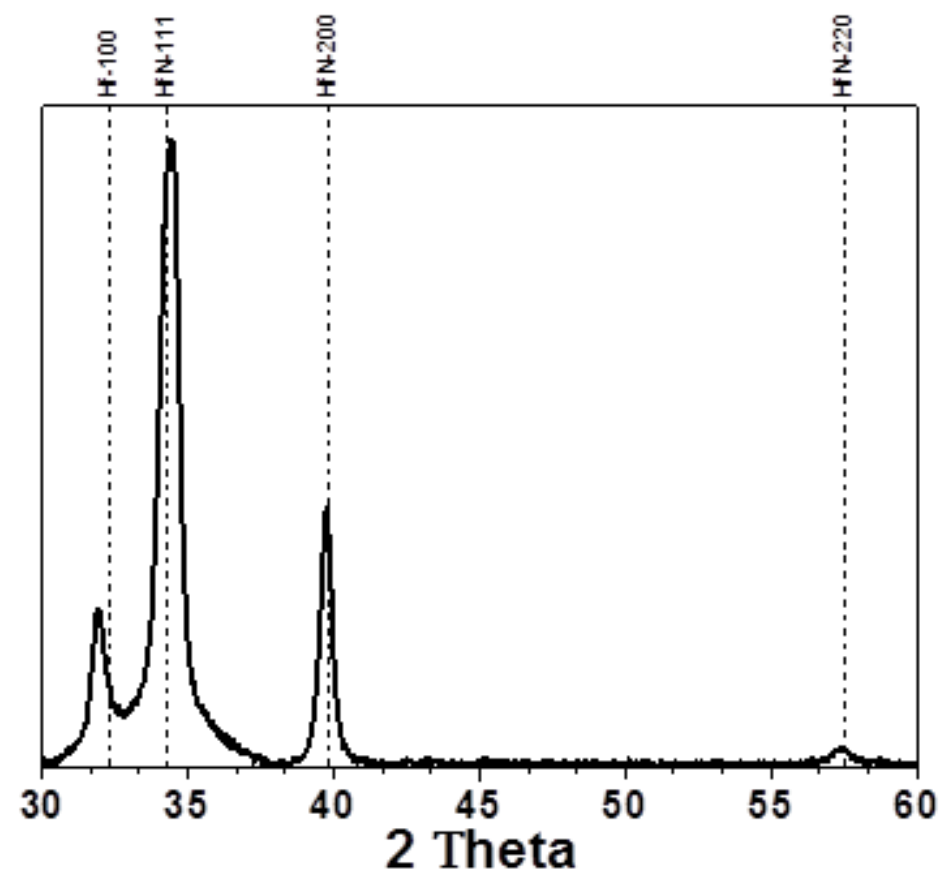

Fig. 4: Patrón de difracción de rayos $\mathrm{X}$, correspondiente al recubrimiento de nitruro de hafnio.

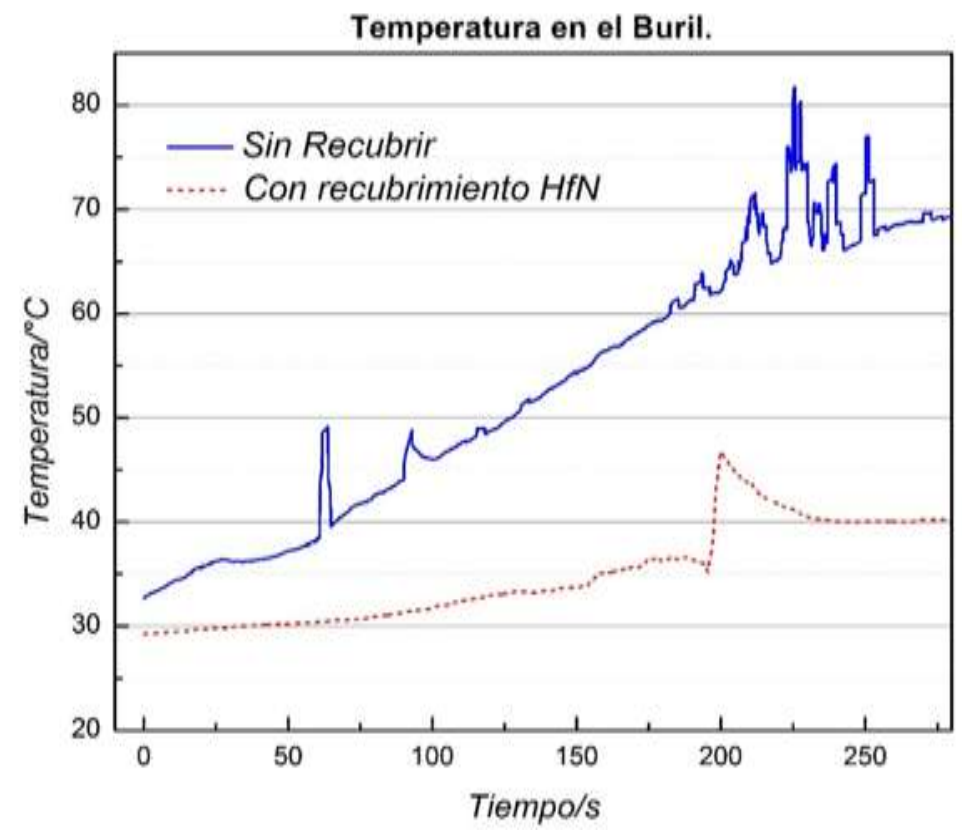

Fig. 5: Temperatura en las Herramientas de corte, con y sin recubrimiento monocapa de HfN, en el cilindrado de probetas de acero AISI1020. 
Tabla 4: Medición de la temperatura en el Buril durante el cilindrado de probetas de acero AISI1020.

\begin{tabular}{|l|l|l|}
\hline Parámetros & Buril sin recubrimiento & Buril recubierto con HfN. \\
\hline Promedio $\left({ }^{\circ} \mathrm{C}\right)$ & 53,25 & 35,41 \\
\hline Cambios de temperatura $\left({ }^{\circ} \mathrm{C} / \mathrm{min}\right)$ & 7,41 & 2,711 \\
\hline Temperatura de Estabilización $\left({ }^{\circ} \mathrm{C}\right)$ & 68 & 40 \\
\hline Temperatura Máxima. $\left({ }^{\circ} \mathrm{C}\right)$ & 81,71 & 48,05 \\
\hline
\end{tabular}

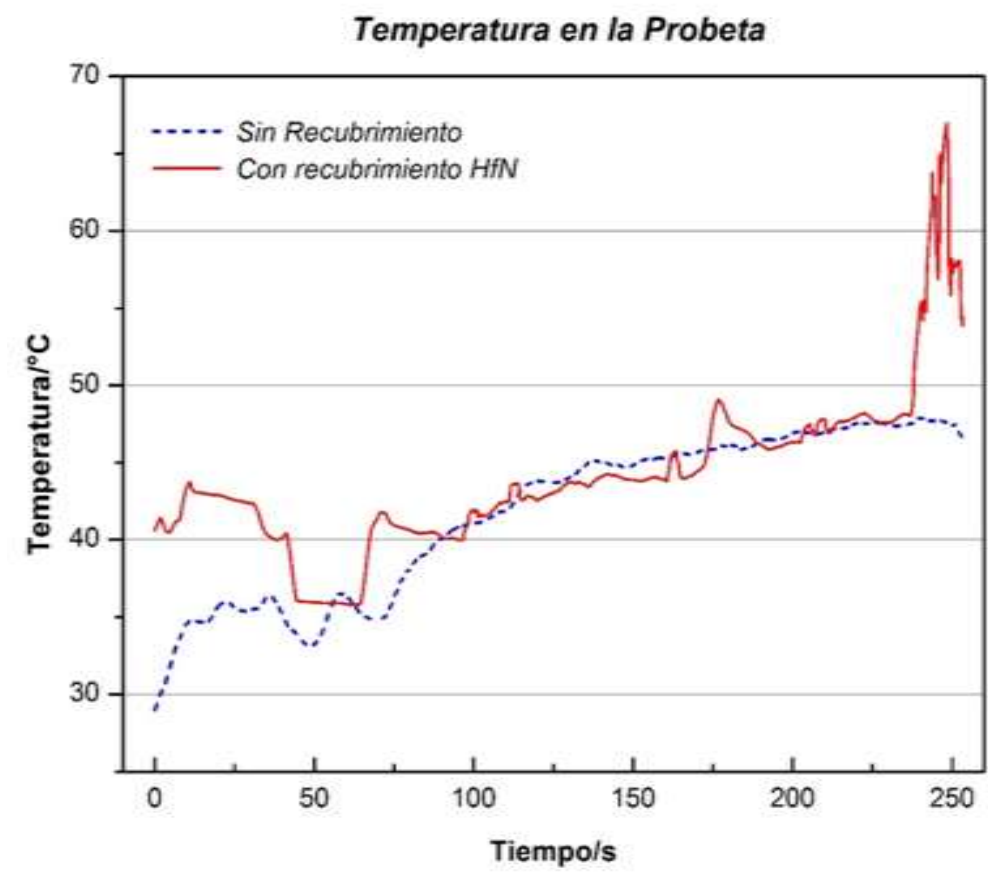

Fig. 6: Temperatura en la probeta de acero AISI1020, durante el mecanizado con buriles, con y sin recubrimiento monocapa de HfN.

Tabla 5: Medición de la temperatura en el Buril durante el cilindrado de probetas de acero AISI1020.

\begin{tabular}{|l|l|l|}
\hline Parámetros & $\begin{array}{l}\text { Buril sin } \\
\text { recubrimiento }\end{array}$ & $\begin{array}{l}\text { Buril recubierto } \\
\text { con HfN. }\end{array}$ \\
\hline Promedio $\left({ }^{\circ} \mathrm{C}\right)$ & 41,82 & 44,04 \\
\hline Cambios de temperatura $\left({ }^{\circ} \mathrm{C} / \mathrm{min}\right)$ & 3,85 & 3,29 \\
\hline Temperatura de Estabilización $\left({ }^{\circ} \mathrm{C}\right)$ & 47,93 & 66,98 \\
\hline Temperatura Máxima. $\left({ }^{\circ} \mathrm{C}\right)$ & 48 & 55 \\
\hline
\end{tabular}

En la probeta de acero se observa un incremento de la temperatura promedio al ser mecanizada con la herramienta recubierta, esto indica que con el recubrimiento, no solo se disminuye la energía absorbida por la herramienta, ya que parte de esta energía es transmitida al metal mecanizado. Aun así, con la herramienta recubierta, se reduce la tasa de cambio de la temperatura, disminuyendo la deformación térmica y esfuerzos internos generados en la pieza mecanizada durante el proceso de corte. Con la herramienta recubierta aumenta el trabajo efectuado sobre la pieza; por lo cual la temperatura de estabilización aumento de $48^{\circ} \mathrm{C}$ a $55^{\circ} \mathrm{C}$, lo cual produce un mejor acabado superficial de la pieza, sin haber modificado los parámetros del corte. El incremento de la temperatura máxima de la probeta durante el corte, al utilizarse el buril recubierto, indica que la herramienta puede generar mayor desprendimiento del material sin requerir variar el área de contacto con la pieza o las velocidades del corte. 
Temperatura en la Viruta

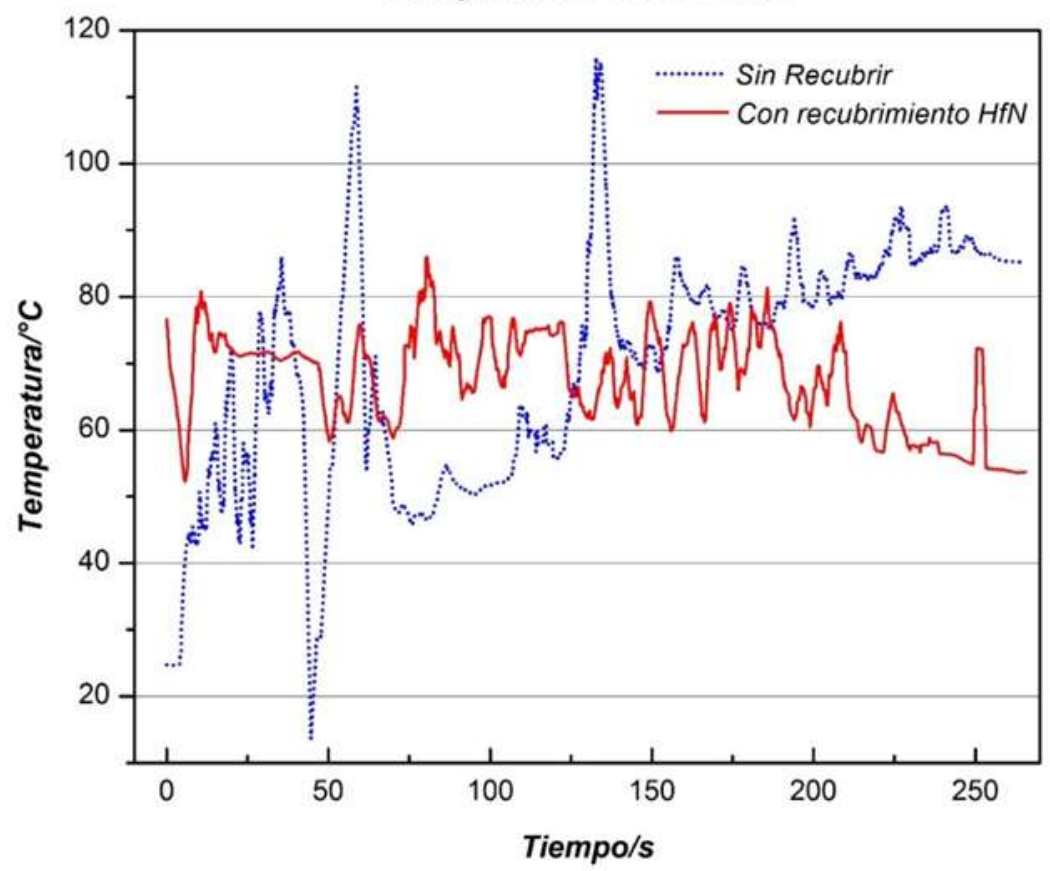

Fig. 7: Temperatura en la viruta, durante el mecanizado del acero AISI1020, con buriles, con y sin recubrimiento monocapa de HfN.

Tabla 6: Medición de la temperatura en la viruta, durante el cilindrado de probetas de acero AISI1020.

\begin{tabular}{|l|l|l|}
\hline Parámetros & $\begin{array}{l}\text { Buril sin } \\
\text { recubrimiento }\end{array}$ & $\begin{array}{l}\text { Buril recubierto } \\
\text { con HfN }\end{array}$ \\
\hline Promedio $\left({ }^{\circ} \mathrm{C}\right)$ & 69,95 & 67.44 \\
\hline Cambios de temperatura $\left({ }^{\circ} \mathrm{C} / \mathrm{min}\right)$ & 13,69 & $-5,19$ \\
\hline Temperatura Máxima $\left({ }^{\circ} \mathrm{C}\right)$ & 115,80 & 86,02 \\
\hline Temperatura de Estabilización. $\left({ }^{\circ} \mathrm{C}\right)$ & 87 & 55 \\
\hline
\end{tabular}

La viruta arrancada con la herramienta recubierta reduce su temperatura promedio, reflejando que la energía se disipa en la probeta mecanizada, y no se disipada en el material desprendido. Se reduce de $87^{\circ} \mathrm{C}$ a $55^{\circ} \mathrm{C}$ la temperatura de estabilización, revelando que se requiere menor trabajo para generar el arranque de material durante el corte; y la temperatura máxima se redujo en $29^{\circ} \mathrm{C}$ reflejando mayor aprovechamiento de la energía producida en el corte del acero. También se presenta una reducción de la tasa de cambio de temperatura, debido a que con la herramienta recubierta las temperaturas más altas se presentan en los instantes iniciales del corte, contrario a lo que sucede con el buril convencional (sin recubrir); que indica mejor estabilidad del corte generado, aumento en la uniformidad del arranque de viruta, y en consecuencia mejor acabado superficial de la pieza.

\section{Rugosidad y acabado superficial}

En la figura 8 se observan las varillas mecanizadas de acero AISI 1020, luego de realizar el pulido, con buriles con y sin recubrimiento, la metodología desarrollada se indica en los detalles experimentales. Se observa que para el ensayo con el buril sin recubrir se generó viruta continua con recrecimiento del filo, debido a que la fricción entre la viruta y la herramienta es alta para que la viruta se acople a la cara del buril, la presencia de este material adherido aumenta más la fricción, y esto genera la autosoldadura, generando un aumento de la rugosidad superficial. Con respecto a los ensayos realizados con los buriles recubiertos, se generó viruta tipo continua, la energía disipada que se transmiten a la viruta en la interfase existente entre ella y la herramienta de corte, debido a esto se logra un buen acabado superficial, con un bajo valor de rugosidad (Escobar et al., 2014 y Szymańska et al., 2014). En la tabla 7, se evidencia que al usar la herramienta recubierta se presenta mejor acabado superficial, al reducir la irregularidad de la pieza trabajada; todo esto reflejado en el cambio de la temperatura del proceso en función del tipo de herramienta utilizada. 


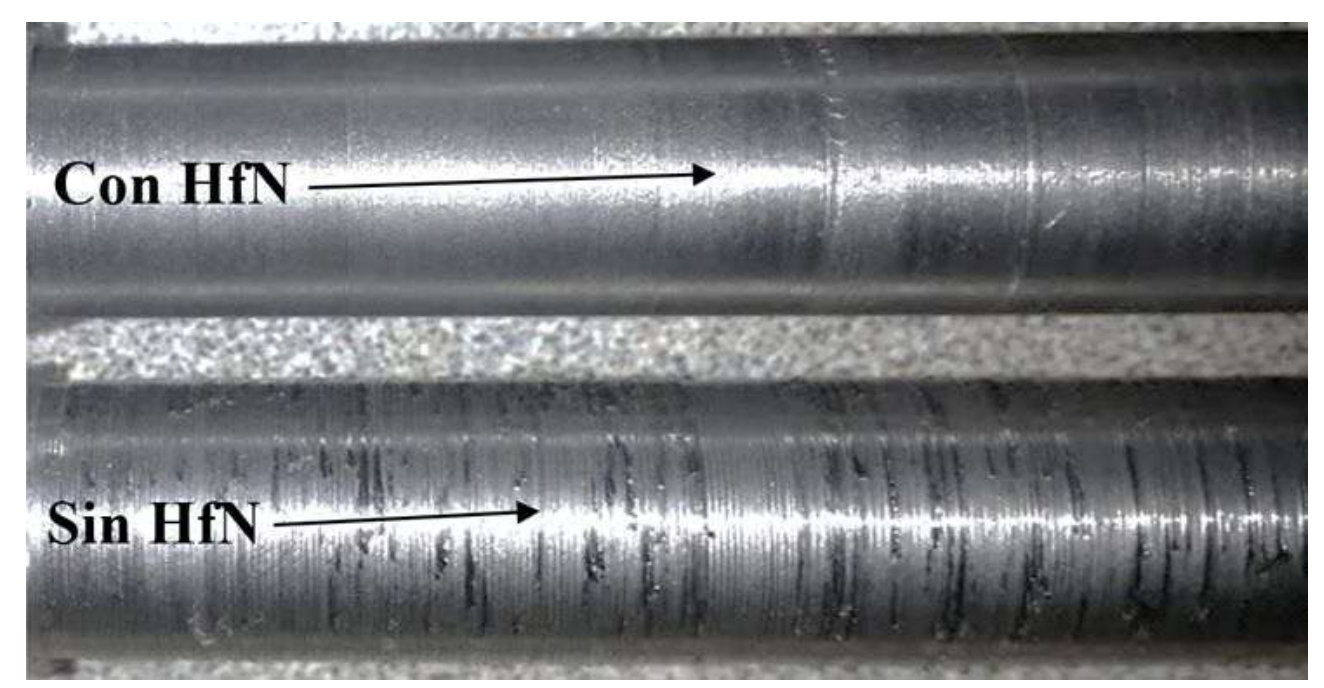

Fig. 8: Probetas de acero AISI1020 obtenidas luego del pulido al utilizar buriles, con y sin recubrimiento monocapa de $\mathrm{HfN}$.

Tabla 7: Rugosidades en probetas de acero pulidas con herramienta recubierta y sin recubrimiento de HfN.

\begin{tabular}{|l|l|l|l|}
\hline Característica & Rugosidad Promedio $(\mu \mathrm{m})$ & Rugosidad Máxima $(\mu \mathrm{m})$ & Rugosidad mínima $(\mu \mathrm{m})$ \\
\hline Sin recubrimiento & 7,61 & 18,3 & 2,46 \\
\hline Recubrimiento HfN & 5,0 & 8,16 & 2,22 \\
\hline
\end{tabular}

\section{Caracterización mediante MEB}

En las figuras 9 a y $\mathrm{b}$, se presenta la caracterización morfológica realizada mediante microscopia electrónica de barrido (MEB) de los buriles sin y con recubrimientos, luego del proceso de cilindrado. En todos los materiales se evidenciaron mecanismos de desgaste de flanco, el cual se presenta en la superficie de incidencia de la herramienta y es atribuido generalmente al frotamiento de la herramienta sobre la superficie mecanizada y a las temperaturas de trabajo (Álvarez, 2009). En la figura 9 se observa que los materiales que presentaron mayor desgaste fueron el buril sin recubrimiento (Figura 9a), seguidos por el buril recubierto con HfN (Figura 9b). El mejor comportamiento frente al desgaste luego del proceso de mecanizado estuvo dado por el buril recubierto con $\mathrm{HfN}$, donde se observan pocas evidencias de desgaste de la herramienta. Este comportamiento del nitruro de Hafnio puede asociarse a la formación de óxido de Hafnio, la cual actúa como lubricante entre las partes en contacto disminuyendo el coeficiente de fricción y a su vez el desgaste (Qiu, Li, et al., 2013).

En la figura 10 se presentan imágenes de MEB, de los buriles con y sin recubrimiento, luego de haber realizado los procesos de cilindrado y posterior pulidos o rectificados de las muestras cilindradas. Se observa disminución del filo de la herramienta después del pulido, así como también desgaste del flanco manifestado como desprendimiento del material (Figuras 10a y 10b). El buril recubierto con HfN presentó un mejor comportamiento al desgaste en los procesos de cilindrado y pulido (Figura 10b), lo cual se puede atribuir al buen comportamiento frente al desgaste que presenta el HfN, como ya se había mencionado la formación de óxidos de hafnio cumplen una función lubricante cuando se pone en contacto con el material a maquinar, disminuyendo la probabilidad de presentar mecanismos de desgaste en la herramienta (Qiu, Leed, et al., 2013; y Zhoua et al., 2010).

En resumen todo lo anterior indica, a partir de la medición de las temperaturas, que con el uso del recubrimiento monocapa $\mathrm{HfN}$, se presenta menor transferencia de energía a la herramienta, y así el desgaste, sufrido por la herramienta será menor; además la energía generada en el corte es transmitida en mayor grado al material metálico mecanizado. Incluso se presenta una mayor estabilidad de la transferencia de energía entre los elementos involucrados; y de acuerdo a lo expuesto por Davies et al. (2007), a menor tasa de cambio de temperatura, es mejor la calidad del proceso de corte y el acabado de la pieza.

\section{CONCLUSIONES}

A partir de los resultados obtenidos, su análisis y discusión, se evidencia que la deposición de recubrimientos monocapa de $\mathrm{HfN}$ en buriles de acero rápido disminuyen el desgaste y pueden aumentar la vida útil de la herramienta, mejorar la calidad del producto manufacturado, disminuir los tiempos y costos de producción; por lo cual su aplicación podría ofrecer distintas ventajas a nivel industrial. 


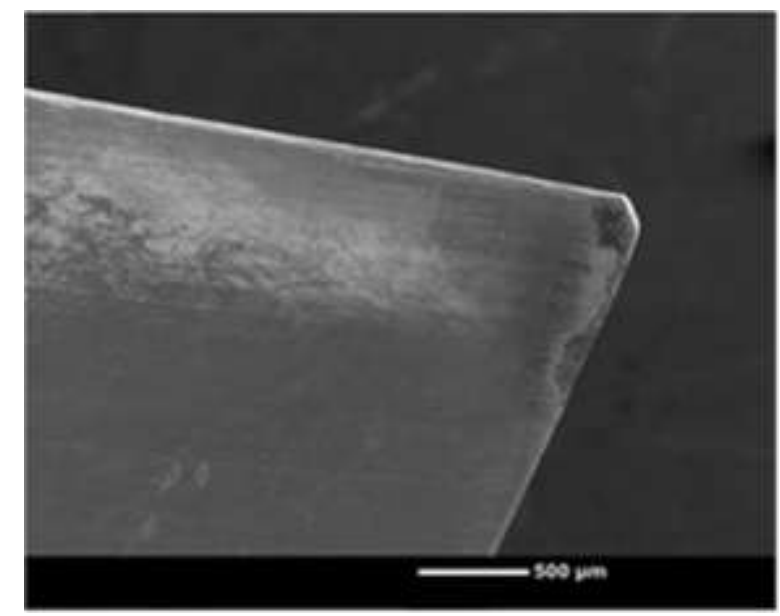

(a)

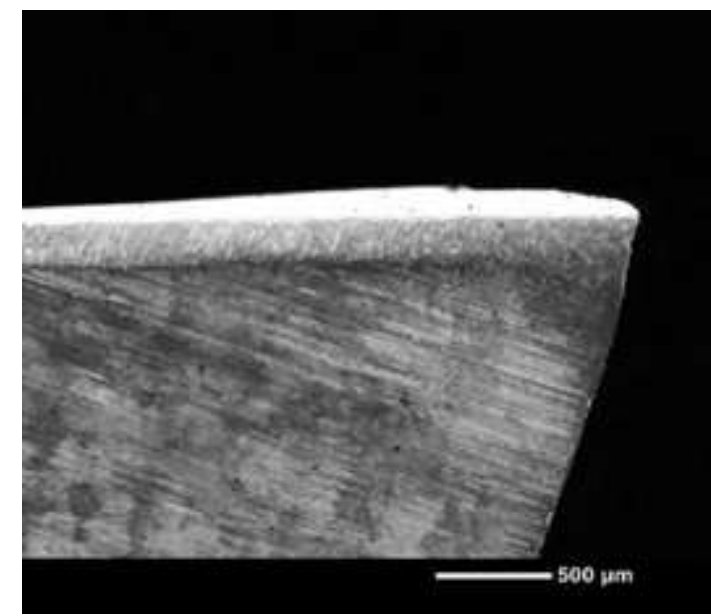

(b)

Fig. 9: Micrografías de SEM de los buriles luego del proceso de cilindrado: a) Buril sin recubrir; b) Buril recubierto con HfN.

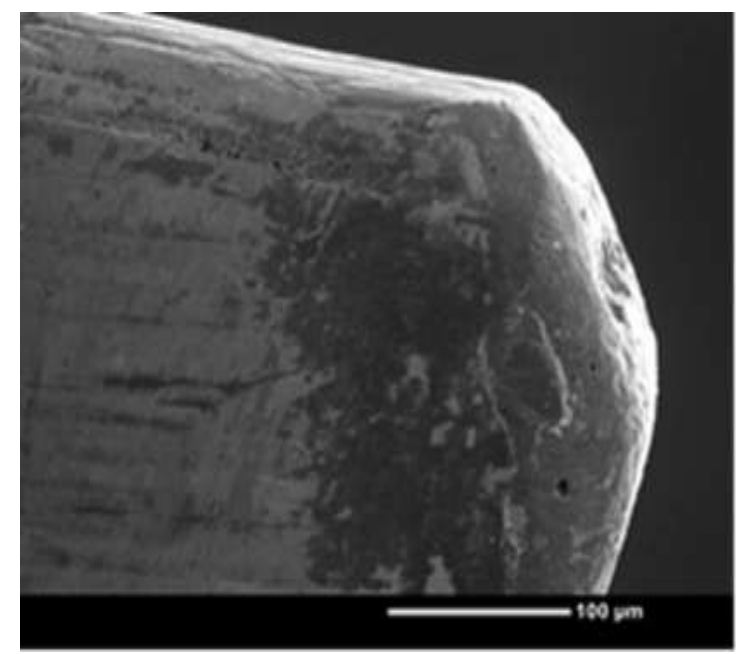

(a)

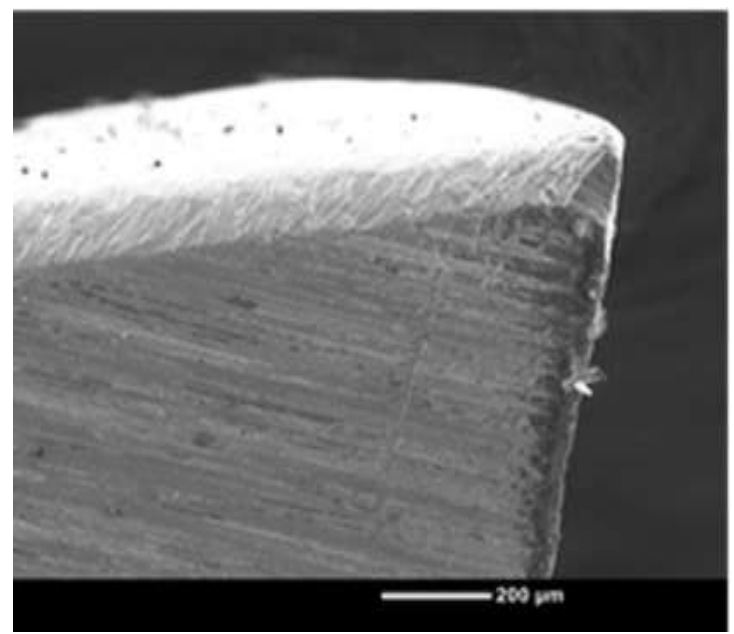

(b)

Fig. 10: Micrografías de SEM del buril recubierto con HfN. Comparación de los mecanismos de desgaste luego de los procesos de cilindrado y pulido.

\section{AGRADECIMIENTOS}

Los autores agradecen a la Vicerrectoría de Investigaciones de la Universidad Militar Nueva Granada por la financiación de este trabajo, derivado del proyecto ING1775 vigencia 2015.

\section{REFERENCIAS}

Álvarez I. R., "Análisis por el método de elementos finitos de modelo simplificado de corte en aleaciones ligeras de interés Aeroespacial". Tesis doctoral. Universidad nacional de educación a distancia, Departamento de Ingeniería de Construcción, España (2009)

Alves M.L., Ferreira B.B., Leta F.R., "Evaluación de Parámetros de Rugosidad usando Análisis de Imágenes de Diferentes Microscopios Ópticos y Electrónicos", Información Tecnológica, 22 (4), 129-149 (2011)

Åstrand M., Selinder T.I., Fietzke F., Klostermann H., "PVD-Al2O3-coated cemented carbide cutting tools", Surface and Coatings Technology, Volumes 188-189, 186-192 (2004)

Beake B.D., Fox-Rabinovich G.S., "Progress in high temperature nanomechanical testing of coatings for optimising their performance in high speed machining", Surface and Coatings Technology, 255, 102-111 (2014) 
Bobzin K., Bagcivan N., Ewering M., Brugnara R.H., Basturk S., "Comparison of (Ti,Al)N and (Ti,Al)N/YAl2O3 coatings regarding tribological behavior and machining performance", Surface and Coatings Technology, 257, 58-62 (2014)

Bouzakis K.-D., Skordaris G., Gerardis S., Katirtzoglou G., Makrimallakis S., Pappa M., Lill E., M'Saoubi R., "Ambient and elevated temperature properties of TiN, TiAIN and TiSiN PVD films and their impact on the cutting performance of coated carbide tools", Surface and Coatings Technology, 204 (6-7), 1061-1065 (2009)

Capote Rodriguez, G., Capote Mastrapa, G., y Olaya, J. "Resistencia al desgaste y a la corrosión de recubrimientos de dlc depositados sobre aceros AISI 304 y AISI 1020", Revista Latinoamericana de Metalurgia y Materiales, 35(1), 134-141 (2015)

Davies M.A., Ueda T., M'Saoubi R., Mullany B., Cooke A.L., "On The Measurement of Temperature in Material Removal Processes”, CIRP Annals - Manufacturing Technology, 56 (2), 581-604 (2007)

El Hakim M.A., Shalaby M.A., Veldhuis S.C., Dosbaeva G.K., "Effect of secondary hardening on cutting forces, cutting temperature, and tool wear in hard turning of high alloy toolsteels", Measurement, 65, 233-238 (2015)

Escobar C.A., Caicedo J.C., Aperador W., "Corrosion resistant surface for vanadium nitride and hafnium nitride layers as function of grain size", Journal of Physics and Chemistry of Solids, 75 (1), 23-30 (2014)

Kant G., Sangwan K. S., "Prediction and optimization of machining parameters for minimizing power consumption and surface roughness in machining", Journal of Cleaner Production, 83, 151-164 (2014)

Klocke F., Gerschwiler K., Fritsch R., Lung D., "PVD-coated tools and native ester - an advanced system for environmentally friendly machining", Surface and Coatings Technology, 201 (7), 4389-4394 (2006)

Mandal N., Doloi B., Mondal B., "Development of flank wear prediction model of Zirconia Toughened Alumina (ZTA) cutting tool using response surface methodology", International Journal of Refractory Metals and Hard Materials, 29(2), 273-280 (2011)

Nayak Sh. K., Patro J. K., Dewangan S., Gangopadhyay S., "Multi-objective Optimization of Machining Parameters During Dry Turning of AISI 304 Austenitic Stainless Steel Using Grey Relational Analysis". Procedia Materials Science, 6, 701-708 (2014)

Nieto J. E., Moreno Chávez L.H., Amaya Hoyos C.A.., "Aplicación de recubrimientos multicapa para el mejoramiento de la productividad de las empresas". Informador Técnico, 74, 57-63 (2010)

Palanikumar K., Davim J., "Mathematical model to predict tool wear on the machining of glass fibre reinforced plastic composites", Materials \& Design, 28 (7), 2008-2014 (2007)

Pawade R.S., Joshi Suhas S., Brahmankar P.K., "Effect of machining parameters and cutting edge geometry on surface integrity of high-speed turned Inconel 718", International Journal of Machine Tools and Manufacture, 48 (1), 15-28 (2008)

Qiu Y., Zhang S., Leed J., Li B., Wang Y., Zhao D. "Self-lubricating CrAIN/VN multilayer coatings at room temperature". Applied Surface Science, 279, 189-196 (2013)

Qiu Y., Zhang S., Li B., Wang Y., Lee Y., Li F., Zhao D. "Improvement of tribological performance of CrN coating via multilayering with VN". Surface \& Coatings Technology", 231, 357-363 (2013)

Sai Chaitanya Kishore D., Prahlada Rao K., Mahamani A., "Investigation of Cutting Force, Surface Roughness and Flank Wear in Turning of In-situ Al6061-TiC Metal Matrix Composite", Procedia Materials Science, 6, 1040-1050 (2014)

Smith I.J., Gillibrand D., Brooks J.S., Münz W.-D., Harvey S., Goodwin R., "Dry cutting performance of HSS twist drills coated with improved TiAIN", Surface and Coatings Technology, 90(1-2), 164-171 (1997)

Staia M., Bhat D., Puchi-Cabrera E., Bost J., "Characterization of chemical vapor deposited HfN multilayer coatings on cemented carbide cutting tools", Wear, 261 (5-6), 540-548 (2006) 
Szymańska M., Gierałtowska S., Wachnicki Ł., Grobelny M., Makowska K., Mroczyński R., "Effect of reactive magnetron sputtering parameters on structural and electrical properties of hafnium oxide thin films", Applied Surface Science, 301, 28-33 (2014)

Vogtel P., Klocke F., Lung D., "High Performance Machining of Profiled Slots in Nickel-Based-Superalloys", Procedia CIRP, 14, 54-59 (2014)

Young H., "Cutting temperature responses to flank wear", Wear, 201(1-2), 117-120 (1996)

Zhoua Z., Rainfortha W.M., Luob Q., Hovsepianb P.Eh., Ojedac J.J., Romero-Gonzalez M.E. "Wear and friction of TiAIN/VN coatings against Al2O3 in air at room and elevated temperatures". Acta Materialia, 58, 2912-2925 (2010) 
